\title{
Estudio sobre la contribución de la radio al envejeci- miento activo. Fase inicial: análisis cuantitativo de la presencia de la vejez en los magazines de radio $^{1}$
}

\author{
Hugo AzNAR \\ Universidad CEU Cardenal Herrera \\ haznar@uch.ceu.es \\ Ángels Álvarez Villa \\ Universidad CEU Cardenal Herrera \\ aalvarez@uch.ceu.es \\ Amparo SUAY MADRID \\ Fundación Salud y Comunidad (FSC) \\ amparo_suay_mad@hotmail.com
}

Recibido 7 de abril de 2015

Aceptado: 20 de octubre de 2015

\section{Resumen}

Este trabajo aborda la posible contribución de la radio al envejecimiento activo. La ética comunicativa actual debe tener presentes los colectivos más vulnerables de la sociedad y uno de ellos es el de la vejez. Se recogen en el artículo los principales datos demográficos sobre población mayor, cuyo crecimiento es tal que se hace necesario un cambio de paradigma a la hora de abordar estos cambios, lo que se conoce como envejecimiento activo. Se plantea que la radio, por sus peculiares características, puede contribuir a promover dicho paradigma. Pero es necesario partir de datos objetivos y el artículo concluye presentando los datos y gráficos de la investigación realizada sobre la presencia de la vejez en los magazines matinales de las cuatro emisoras más importantes del país.

Palabras clave: Ética de la Comunicación, Públicos vulnerables, Radio, Magazines de radio, Envejecimiento Activo.

\section{Study on the Contribution of the Radio to Active Ageing. Initial Phase: Quantitative Analysis of the Topic of Ageing on Radio Magazines}

\begin{abstract}
This paper deals with the possible contribution of the radio to active ageing. New communicative ethics must take into account the most vulnerable groups of the society and one of them is that of elderly people. The main demographics data of older people are collected in the article and their growth makes clear that is necessary a paradigm shift in addressing these changes, what is already known as active aging. It is stated that the radio, due to its peculiar characteristics, can contribute to promote this paradigm. But in order to make clear how, it is necessary previously to begin with objective data about the real presence of aging in the media. So the article concludes by presenting data and graphics of the research conducted on the presence of old age in the morning magazines of the four major Spanish radio stations.
\end{abstract}

Keywords: Media ethics, Vulnerable Audiences, Radio, Radio Magazines, Active Ageing.

\section{Referencia normalizada}

AZNAR, Hugo; ÁLVAREZ VILLA, Ángels; y SUAY MADRID, Amparo: "Estudio sobre la contribución de la radio al envejecimiento activo. Fase inicial: análisis cuantitativo de la presencia de la vejez en

1 El presente trabajo forma parte de la Línea de Investigación Democracia deliberativa, comunicación y ciudadanía, financiada, entre otras, mediante los fondos internos Indi-14/01 de la U. CEU Cardenal Herrera. Pueden verse las realizaciones de la Línea en su blog: http://blog.uchceu.es/delibecracia/ 
los magazines de radio". Estudios sobre el Mensaje Periodístico. Vol. 22, Núm. 1 (enero-junio), págs.: 143-162. Madrid, Ediciones Complutense.

Sumario: 1. Ampliando la ética de la comunicación: la atención a los colectivos desaventajados. 2. Un reto mediático: una sociedad de personas mayores y un cambio en la concepción de la vejez; 2.1. Relevancia demográfica de la vejez; 2.2. "Envejecimiento del envejecimiento" y "envejecimiento activo". 3. Radio y envejecimiento activo; 3.1. Por qué la radio; 3.2. Objetivo final y fases de análisis. 4. Fase inicial: análisis cuantitativo de la presencia temática de la vejez en magazines de la franja matinal. 5. Conclusiones. 6. Referencias bibliográficas.

\section{Ampliando la ética de la comunicación: la atención a los colectivos desaven- tajados}

Tradicionalmente la ética de la comunicación se había planteado como una serie de normas y deberes ligados a las exigencias básicas de la práctica periodística (como decir la verdad, contrastar la información, cumplir los pactos de confidencialidad, etc.), tal y como se recogían en los primeros códigos éticos del periodismo (Laitila, 1995). Estas exigencias se relacionaban principalmente con la información política nacional, al constituir ésta el núcleo duro de la función tradicional atribuida al periodismo como vertebrador de la esfera pública democrática. Pero conforme los medios han adquirido una posición más central en nuestras sociedades, afectando al conjunto de nuestra vida cultural y social, la anterior visión de la ética comunicativa, con ser básica y seguir desarrollando un papel esencial, se queda corta. Al hilo de la ampliación del papel de los medios, desde los años noventa del siglo pasado se produce una ampliación de los ámbitos abordados por la ética de la comunicación, en un intento de ajustarse al papel 'ampliado' de los medios y a la necesidad de disponer de guías y recomendaciones relativas al tratamiento mediático de 'nuevos' asuntos y ámbitos de interés.

En su día abordamos esta tendencia bajo la rúbrica «ética de la comunicación y nuevos retos sociales» (Aznar, 2005), recalcando el cambio de que la ética comunicativa atendiera al tratamiento de estos nuevos ámbitos informativos, más allá de los mínimos establecidos en la primera generación de códigos deontológicos. Ya en el desarrollo del texto se advertía (Aznar, 2005: 44) que no era tanto que tales asuntos fueran nuevos -pues más bien era al contrario: solían ser los más antiguos o comunes en toda sociedad, como catástrofes, discapacidad, inmigración o violencia de género, por mencionar algunos-, cuanto que sí era nueva su tematización mediática y el consiguiente reto que planteaba a la ética comunicativa su tratamiento correcto.

Lo que está en juego es más bien la apertura del punto de mira de los medios hacia situaciones sociales y colectivos que tradicionalmente estaban excluidos del foco de la atención mediática; o como decía Lippmann ([1920] 2011: 44) en una llamativa metáfora referida al tratamiento de los inmigrantes en la prensa estadounidense de los años veinte: son como los baches de la carretera, que sólo les prestas atención cuando los pisas. Es decir que el periodismo - a riesgo de generalizar-o pasaba de estos temas o cuando no pasaba, era para tratarlos sin ninguna consideración especial o simplemente de manera incorrecta, 'pisando' a los afectados sin reparo alguno.

Dentro de los códigos deontológicos tradicionales, esta ampliación del foco de mira de los medios hacia otros colectivos se planteó inicialmente bajo una óptica de mínimos: estableciendo el deber básico de evitar cualquier forma de discriminación 
en su tratamiento periodístico. Un buen ejemplo tanto del cambio histórico de la sensibilización hacia a estas cuestiones como de este planteamiento mínimo de los códigos tradicionales sería el de la Declaración de principios de la conducta de los periodistas de la IFJ, que se aprobó inicialmente en 1954 y cuyo único cambio posterior fue la inclusión en 1986 de un artículo destinado a evitar cualquier forma de discriminación periodística de las personas o colectivos más vulnerables. Se trataba del artículo 7: «El periodista se cuidará de los riesgos de una discriminación propagada por los medios de comunicación y hará lo posible para evitar que se facilite tal discriminación, fundamentada especialmente en la raza, el sexo, la moral sexual, las opiniones políticas y demás, así como el origen nacional o social» (recogido en Aznar, 2000: 170).

Sin embargo pronto se vio que este deber negativo -este mínimo ético consistente en evitar los daños de un mal periodismo- con ser fundamental, no era suficiente. Para realizar un periodismo verdaderamente ético y comprometido era necesario plantearse también cómo contribuir desde los medios a un tratamiento correcto e incluso positivo de estas cuestiones, que pudiera contribuir a la mejora de la posición social de estos colectivos. Al plantear estas cuestiones era evidente (a) que los medios no eran, por supuesto, los responsables de tales problemas; ni tampoco (b) los únicos llamados a solucionarlos (Aznar, 2005: 52 y ss.); pero también (c) que su papel resultaba crucial para incrementar su percepción social, situarlos en la agenda pública -mediante un periodismo cívico y comprometido- e incluso contribuir de algún modo a la mejora de su situación -mediante un periodismo de soluciones y de servicio-.

Para este enfoque ampliado de la ética comunicación resultan cruciales tres dimensiones interrelacionadas:

i) Vulnerabilidad. Esta noción trata de sustituir a la más restrictiva de discriminación. En efecto, no todos estos colectivos sufren una discriminación por parte de la sociedad: en no pocas ocasiones se trata más bien de situaciones de desconocimiento o ignorancia, aspecto en el que precisamente los medios pueden ayudar mucho a cambiar las cosas. La noción de vulnerabilidad incide en que suele tratarse de grupos en una situación comparativa de desventaja o debilidad frente a las oportunidades y ventajas de que disponen quienes podemos considerar miembros estándar de la sociedad (UNESCO, 2013: 37). Posición de desventaja mucho mayor aún si los comparamos con quienes ocupan mayoritariamente el espacio de los medios que en una cultura mediática del éxito, la fama, el glamour o la buena presencia, suelen ser personas privilegiadas en algún aspecto físico, político, económico, etc. Su posición de desventaja y/o debilidad los hace especialmente vulnerables a un tratamiento incorrecto o a un no-tratamiento por parte de los medios.

ii) Empoderamiento. Se trata ya de una acción mediática proactiva. No es evitar hacer algo -algo negativo o perjudicial-, sino hacer algo positivo: contribuir a que estos colectivos mejoren su situación de desventaja social. Esto requiere contribuir a que quienes integran estos colectivos dispongan de una mayor capacidad: a) de acción autónoma; y b) de influencia en la agenda pública.

Para ello es esencial un primer elemento en el que la contribución de los medios resulta esencial: su visibilización -de ellos y de su posible situación de desventaja-, 
así como la consiguiente oportunidad de dotarles de voz y presencia mediáticas propias. Esta presencia es requisito sine qua non para dar a conocer su situación y dotarles así de fuerza de expresión en la esfera pública mediática, a partir de la cual influir en la agenda pública colectiva.

La otra contribución será la de practicar un periodismo de servicio o de soluciones que ayude a estos colectivos a mejorar su posición, trasladando a su vida personal y su ámbito de actuación aquellas propuestas y soluciones útiles de las que han tenido conocimiento a través de la actividad informativa de los medios ${ }^{2}$. La contribución a dotar de mayor autonomía a estos colectivos pasa hoy en día también por el propio uso y disfrute de los medios. Así, contribuir a su mejora debe facilitar pautas de uso, herramientas e indicaciones acerca de cómo actuar proactivamente en relación a los propios medios de comunicación. En este punto ambos aspectos del empoderamiento se retroalimentan: una mayor capacidad de acción en relación a los medios contribuye a conseguir una mayor visibilización en ellos, lo que dota de fuerza para modificar la agenda pública con el fin de promover medias públicas y sociales que mejoren su situación real.

iii) Ciudadanía (mediática). Es claro por tanto el papel instrumental que en ambos sentidos pueden desempeñar los medios y la práctica de un buen periodismo, de un periodismo ético guiado por pautas de actuación explícitas en este sentido. El resultado será contribuir así a una ciudadanía mediática más equitativa y participativa para todos, a un entorno mediático por ello mismo más justo y equilibrado; una precondición casi imprescindible en el mundo de hoy para la mejora efectiva de la posición en la realidad misma.

\section{Un reto mediático: una sociedad de personas mayores y un cambio en la con- cepción de la vejez}

Uno de los ámbitos que en esta ampliación del foco temático de una comunicación ética no ha recibido hasta ahora la atención debida es el de la tercera edad o la vejez en general. Esta afirmación se plantea a partir de tres factores comparativos:

a) desde la ética comunicativa: si comparamos las recomendaciones éticas sobre el tratamiento mediático de la vejez aparecidas en los últimos años (AAVV, 2011; EAPN, 2012) con otros ámbitos temáticos en los que ha habido una notable aportación de iniciativas, como en el tratamiento mediático de la violencia del género, la protección de la infancia y otros tantos más;

b) desde la propia relevancia demográfica de la vejez: si tenemos en cuenta que se trata de un colectivo cada vez más numeroso, con un peso social, económico, etc., creciente;

${ }^{2}$ Conviene recordar respecto al periodismo de soluciones, para evitar una confusión común, que no se trata de que los medios encuentren la solución a estos problemas (la mediatización de nuestras sociedades tiende a proyectar equivocadamente esta exigencia sobre los medios); sino de que informen de las soluciones que han podido darse a esos mismos problemas en otros lugares, dándolas así a conocer y facilitando su difusión a otros entornos (Aznar, 2005: 56; Bornstein, 2011). 
c) desde su consiguiente relevancia para el consumo y el contenido de los medios (y de la publicidad), especialmente, como luego destacaremos, en algunos soportes como la radio.

\subsection{Relevancia demográfica de la vejez}

El envejecimiento de la población es un fenómeno internacional con grandes repercusiones sanitarias, sociales, económicas y culturales. En las sociedades occidentales se produce un envejecimiento acelerado que hace que el colectivo de personas mayores esté creciendo a un ritmo mayor que el resto de grupos de población. En este sentido, "la primera tendencia emergente en España, al igual que en otros países europeos, es la inversión demográfica. Es decir, por primera vez en la historia, las personas de 65 y más años superan a los niños de $0-14$. El conjunto de la población está envejeciendo" (IMSERSO, 2011: 41).

En el informe del IMSERSO coordinado por Rosa Díaz (2012) también se presentan otros indicadores demográficos que proporcionan una visión sobre el proceso de envejecimiento a nivel mundial, siempre mirando al futuro. En nuestro caso, este fenómeno se revela especialmente acelerado, siendo España uno de los países con mayor número de personas mayores:

Entre el año 2010 y el 2050 se estima que la población mundial de 65 años y más se multiplicará por tres, pasando de 523 millones a 1.486 millones. De acuerdo con las proyecciones de población de Naciones Unidas, en el año 2050 el 26,2\% de la población de los países desarrollados superará los 65 años y el 14,6\% en los países en vías de desarro1lo. La comparación mundial del proceso de envejecimiento mundial posiciona a España entre los países más envejecidos del planeta (17,2\% de población de 65 años y más en 2010). Japón encabeza el ranking de países con mayor proporción de población mayor (22,6\%). (Díaz, 2012: 32).

España es uno de los países europeos con una cifra más alta de personas mayores, junto con Alemania, Francia, Reino Unido e Italia, como corresponde con el hecho de ser los países más poblados. Así, según los indicadores estadísticos más recientes, "a 1 de enero de 2014 hay 8.442 .427 personas mayores (65 y más años), el 18,1\% sobre el total de la población (46.771.341), según los datos del Padrón Continuo (INE). Sigue creciendo en mayor medida la proporción de octogenarios; ahora representan el 5,7\% de toda la población" (Abellán y Pujol, 2015: 3).

El porcentaje de población mayor de 65 años, que actualmente se sitúa en el 18,2\%, pasará a ser el $24,9 \%$ en 2029 y el 38,7\% en 2064, según datos del INE. Dentro de 15 años, en España residirán 11,3 millones de personas mayores de 64 años, 2,9 millones más que en la actualidad. Y esta cifra se incrementará hasta 15,8 millones de personas en 50 años, según la misma fuente (INE, 2014b).

En cuanto a las causas del envejecimiento de la población española destaca el gran aumento de la esperanza de vida, que no se compensa con más nacimientos. Además, el aporte de la inmigración exterior se reduce con la crisis, y por contra los españoles que emigran al exterior han vuelto a aumentar estos últimos años. Así pues, "la mejora de la sanidad y las condiciones sociales y económicas han disparado la esperanza de 
vida en muy poco tiempo hasta convertirla en una de las más altas de Europa" (Carbajosa, 2014: 2). De acuerdo con las últimas proyecciones del INE, las muertes superarán ya este año 2015 a los nacimientos en España, dos años antes de lo previsto (íb.).

Las proyecciones para las próximas décadas indican que el porcentaje de personas mayores en nuestra sociedad se incrementará más y más, habiendo llegado a estimar alguno que en el año 2050 España será el país más viejo del mundo, con un $40 \%$ de personas mayores de 60 años. (Fernández-Ballesteros, 2002: 31). El siglo XXI va a ser, sin duda, testigo de una transición demográfica, en la que la población a nivel mundial envejecerá a una velocidad sin precedentes.

\section{2. "Envejecimiento del envejecimiento" $y$ "envejecimiento activo"}

El espectacular aumento de la esperanza de vida-incrementado en un $126 \%$ entre la primera década del siglo XX y el final de esta centuria- se ha visto motivado por el descenso de la mortalidad infantil, acentuado sobre todo en los dos primeros tercios del siglo pasado, y por la ganancia de años de vida en edades avanzadas (Díaz, 2002: 63). Así en 2009 la esperanza de vida al nacer alcanzaba los 84,0 años en los varones y los 88,7 en las mujeres, lo que supone incrementar los valores actuales en 4 y 3 años respectivamente. En 2064, de mantenerse la tendencia actual, la esperanza de vida de los hombres superará los 91 años y la de las mujeres casi alcanzará los 95 (INE, 2014).

Es decir que no sólo se produce un aumento de la proporción de gente mayor sino que además la vejez se prolonga durante más años al aumentar la esperanza media de vida. Es lo que se ha llamado "envejecimiento del envejecimiento", rasgo destacado de las tendencias actuales de nuestra demografía. En este sentido, los octogenarios seguirán creciendo; de hecho su número ya crece a un ritmo mayor que el del resto de grupos de población. En España ya hay 2,2 millones de personas mayores de 80 años y su cifra se ha multiplicado por 19 (i) desde principios del siglo pasado. Se calcula que en 2049 la cifra volverá doblarse sobradamente: serán 5,6 millones y habrán crecido aún más que el resto de las personas de edad (IMSERSO, 2011b: 43). Conforme siga creciendo la longevidad, el concepto de persona muy mayor se modificará; los octogenarios serán más habituales y los centenarios dejarán de ser una excepcionalidad (Giró, 2006; IMSERSO, 2011b).

Para abordar este fenómeno de la prolongación de la vejez - por lo general en una condiciones sociosanitarias mejores, como lo prueba esa misma prolongación, pero cuya mejora de su calidad de vida no deja de plantear un reto- se ha planteado en las últimas décadas un nuevo paradigma de envejecimiento, para el que se han propuesto diversas denominaciones como "envejecimiento saludable", "envejecimiento con éxito" o "envejecimiento activo", término este último que viene siendo el más utilizado. La caracterización de la vejez ha ido evolucionando así desde la definición del envejecimiento saludable -centrado en la salud- hacia un modelo multidimensional que comprende, obviamente, condiciones bio-médicas, pero que también añade un amplio conjunto de determinantes económicos, sociales, personales y comportamentales al considerar la calidad de vida en la vejez.

En esta línea de ampliación del concepto mismo de envejecimiento, el término "envejecimiento activo" fue adoptado por la OMS a finales de los años 90, definién- 
dolo como: "el proceso por el que se optimizan las oportunidades de bienestar físico, social y mental durante toda la vida, con el objetivo de ampliar la esperanza de vida saludable, la productividad y la calidad de vida en la vejez". Es fácil percibir que esta definición no solo contempla el envejecimiento desde la atención sanitaria, sino que incorpora otros tantos factores sociales, económicos y culturales que afectan al envejecimiento de las personas. Con todo ello se busca

"extender la calidad, la productividad y esperanza de vida a edades avanzadas. Además de seguir siendo activo físicamente, es importante permanecer activo social y mentalmente, participando en actividades recreativas, de voluntariado o remuneradas, culturales, sociales y educativas" (Zamarrón, 2007: 7).

En este sentido, los aspectos que inciden en un envejecimiento activo son los hábitos comportamentales saludables y el adecuado funcionamiento cognitivo y emocional de la persona. Pero más allá del bienestar psicosomático -y también como parte de él- el término "activo" apunta a una participación efectiva en asuntos personales, sociales, económicos, políticos.

Esta importancia de la participación en el envejecimiento activo quiso ponerla de manifiesto la Unión Europea al declarar el 2012 Año europeo del Envejecimiento Activo y la Solidaridad entre Generaciones, con acciones en tres áreas: empleo, participación social y fomento de una vida independiente. Este Año Europeo se destaca en el informe del Eurobarómetro de 2012 -sondeo de opinión del Parlamento Europeo entre los ciudadanos europeos- como una ocasión para fomentar las oportunidades de las personas mayores para participar activamente en la sociedad, para cambiar la actitud y las políticas dirigidas a este fin, y para generar las mejores prácticas para alcanzar ese objetivo (Abdullah y Wolbring, 2013: 6801). No en vano en el Artículo 25 de la Carta de Derechos Fundamentales de la UE se señala que "la Unión reconoce $\mathrm{y}$ respeta el derecho de las personas mayores a llevar una vida digna e independiente, y a participar en la vida social y cultural".

\section{Radio y envejecimiento activo}

Para la calidad de vida del colectivo de las personas mayores las necesidades de socialización, relacionadas con los aspectos afectivos, con la necesidad de compañía del ser humano y con la participación social poseen un papel esencial. Por su caracterización funcional y nivel de dependencia, y en función de las capacidades sensoriales, cognitivas y físicas, las exigencias comunicativas, así como las de pertenencia a un grupo, pueden presentarse como aspectos cardinales en su experiencia vital; máxime si el objetivo es su bienestar en sentido amplio. En este sentido los medios de comunicación juegan un papel esencial para este colectivo de edad, lo que debería reflejarse tanto en la presencia temática de esta franja de edad y sus intereses, como en su representación en los medios, que si bien en ocasiones es positiva (Travers y Barlett, 2011), suele ser mayoritariamente negativa en los medios de comunicación (Harwood, 2007). 


\subsection{Por qué la radio}

En nuestra sociedad la radio y la tele, que por sus atributos provocan una más fácil asimilación de sus mensajes, desplazan a la prensa, con un discurso más intelectual y frío (Sartori, 1998: 49; IBV, 2006). En este paradigma audiovisual en el que vivimos, y por lo que respecta a la dieta mediática y cultural de la población mayor de 65 años, este hecho todavía se acentúa más, siendo el consumo televisivo el que ocupa el primer lugar seguido del radiofónico ${ }^{3}$.

Por lo que respecta a la radio, la naturaleza poderosa de lo auditivo está ligada a la combinación en un mismo mensaje de las cuatro funciones clásicas de la comunicación radiofónica: informar, persuadir, formar y entretener (Merayo, 1992), que se fusionan en la oferta programática de un medio caracterizado por su facilidad de acceso. El discurso más emocional y cercano, y su potencial para involucrar al receptor, son el cordón umbilical que mantiene al destinatario del mensaje escuchando al otro lado del canal. "El hábito y la familiaridad de la escucha induce a una actitud comunicativa tal que el radioyente incorpora a su realidad cotidiana la realidad comunicativa que representa el emisor" (Balsebre, 1994: 223).

En el colectivo de mayores el consumo de programación sonora se encuentra vinculada a la radio generalista, a la radio todo-palabra, y no tanto a la radio musical, frente al resto de la población. La oralidad, la voz humana y sus prestaciones y posibilidades comunicativas, han reafirmado a la radio como el medio de la cercanía, de la proximidad afectiva y la complicidad. En ese espacio de la distancia corta, la radio promueve además la ilusión de una relación interpersonal cuasidirecta. Capaz de generar unas relaciones con el oyente intensas, con mayor fuerza emocional, la palabra no tecnologizada resulta más poderosa por su calidez, vitalidad y ausencia de artificialidad. ${ }^{4}$

En la reflexión sobre el espacio acústico y los mayores, la radio destaca esencialmente también por suponer una liberación de la vista. Su estilo conversacional, que emula el hablado, se ha constituido en la principal baza del medio, pues la mente se asienta sobre un soporte de conocimiento dialógico, y a la vez enlaza con la esencia del pensamiento ya que el lenguaje primario es por excelencia el oral. Asimismo, el poder de la radio descansa en la credibilidad de la que goza, lo que se suma a su capacidad de servir de compañía y a su proximidad psicológica.

El medio, además, tiene un largo itinerario interactivo, una enorme capacidad democratizadora y constructora de significados políticos y socioculturales al servicio del ciudadano. La radio "es producción y construcción de sentido y promociona el debate

3 Este target de edad dedica diariamente 4h 40' a ver la televisión (56 minutos más que el conjunto de la población), 1h 10' a escuchar la radio (17 minutos menos), 18 minutos a leer diarios (el mismo tiempo de dedicación), 6 minutos a navegar por internet (39 minutos menos) y 3 minutos a leer revistas (1 minuto menos). El 92\% de la población mayor de 65 años ve la televisión todos los días, sirviéndoles para cubrir tres objetivos: información, ocio y evitar la soledad (recogido en Vera Aranda, 2005).

4 "El lenguaje hablado infunde mayor estimulación al ser escuchado que leído. La palabra hablada ha demostrado también poseer mayor fuerza de convicción que la escrita" (Cebrián Herreros, 1994: 240). 
público. Al participar en ella las audiencias rediseñan sus significados (...) es equivalente a que esté basada en el diálogo, la horizontalidad, la bimulti-direccionalidad y esté orientada al servicio" (Acosta, Badía y Costales, 2014: 49).

La radio ha sido desde sus inicios un medio que ha conseguido establecer un vínculo especial con su audiencia a través de las emociones, la imaginación, las sensaciones, lo que ha cautivado a un público de adultos mayores, que aún hoy siguen manteniendo su 'romance' con la radio. Si entre la tercera edad se detectan determinadas carencias como son un bajo índice asociativo, deficiente o nula información sobre sus derechos, problemas de salud o cierta marginación social, la radio puede constituir una herramienta que contribuya a paliar dichas limitaciones (Colom y Ortes, 2001); y aún mucho más si se adopta una política específica al respecto.

En el momento actual los diferentes agentes socioeducativos afrontan grandes retos para ajustar la educación a las demandas de una sociedad cada vez más compleja y dinámica, y no cambia este reto al hablar de la tercera edad. El Libro blanco del envejecimiento activo del IMSERSO (IMSERSO, 2011) insiste significativamente en este punto de la educación para un envejecimiento activo, rompiendo así el prejuicio habitual que vincula educación e infancia ${ }^{5}$. Y en este terreno, el potencial educativo y social de los medios es inagotable (López Noguero, 2001). En el caso de la radio dicho potencial pasa por ofrecer a los usuarios resortes para desarrollar lecturas críticas, recreación lúdica, para profundizar en sus dotes creativas y, por todo ello, recursos para trazar un camino de desarrollo personal. La radio puede servir para favorecer la recepción de ideas nuevas, de actitudes y sensibilidades, profundizar en la autorrealización, y favorecer la participación. También tiene un enorme potencial en la labor de promover la identidad y la memoria vinculada a grupos sociales relegados o a los que se les niega su capacidad de producción en otros ámbitos de la vida social y cultural (Aguaded, 1994). Así, la tercera edad puede encontrar entre las ondas un discurso útil y atractivo con el que mantener activas sus capacidades e incluso activar otras nuevas.

\subsection{Objetivo final y fases de análisis}

Dada por tanto la importancia creciente de la población de mayores en nuestra sociedad y la relevancia del papel que pueden cumplir los medios, particularmente la radio a la hora de contribuir al envejecimiento activo, se ha procedido a diseñar una investigación sobre esta (posible) contribución suya.

El objetivo final será elaborar una serie de recomendaciones que puedan resultar de utilidad para los medios, más específicamente para la radio, a la hora de promover un tratamiento más ético y positivo del colectivo de personas mayores, contribuyendo así a la ampliación del espectro de la ética de la comunicación y su abordaje del tratamiento de los colectivos más desfavorecidos. Estas recomendaciones deberían tener como objetivo último la promoción del envejecimiento activo, que constituye el norte al que debe encaminarse la mejora de la calidad de vida de la vejez.

${ }^{5}$ Un ejemplo notable de esta demanda educativa de la tercera edad ha sido el éxito de los Programas Universitarios para Mayores, instrumentos útiles para contribuir a la autonomía, la participación, la calidad de vida y... la formación de los mayores. 
Aunque el objetivo último sea la elaboración de estas recomendaciones para un mejor tratamiento comunicativo de la vejez en los medios, y sea por tanto una propuesta de tipo normativo, es obvio que debe partirse primero de un análisis previo del tratamiento efectivo que este tema recibe en los medios, nuevamente en el caso de la radio que es el que nos va a ocupar. En este sentido la investigación comprenderá una serie de Fases:

Fase I) Análisis cuantitativo. Se trata de determinar como punto de partida cuál es el tratamiento del colectivo de mayores en la radio y si realmente existe, como una primera aproximación sugiere, un tratamiento poco visibilizador. Se identifica así una condición de vulnerabilidad mediática del colectivo de los mayores derivada de su escasa presencia temática. Se trata pues de realizar un análisis cuantitativo de dicha presencia y, en la medida de lo posible, compararlo con otras investigaciones similares hechas para este u otro soporte mediático (Harwood, 2007: 150 y ss.; CAC, 2013) para llegar a conclusiones firmes al respecto. Esta primera Fase de la Investigación ya ha sido concluida y sus resultados son los que presentamos más adelante.

Fase II) Análisis cualitativo. En una segunda Fase se realizará un análisis más cualitativo de los contenidos referidos a la tercera edad en la radio, en un doble plano:

II.a) generalista, a partir de los análisis de los programas de radio generalistas y partiendo de los materiales disponibles que han sido ya estudiados en la Fase I, identificando los contenidos relativos a la temática de la tercera edad. Un análisis que deberá ser complementado también con la bibliografía crítica existente, si bien escasa, sobre dicho tratamiento no sólo en radio sino también en otros soportes.

Y II.b) de los programas especializados dirigidos a este colectivo. En relación a estos se hará no sólo un análisis que permita extraer pautas ya experimentadas y puestas en práctica en estos programas sino también se complementará con una metodología de entrevista a sus responsables en los que estos puedan hacer explícita tanto su intención comunicativa, como sus criterios así como sus consideraciones y recomendaciones derivadas de su experiencia profesional ${ }^{6}$.

Este análisis deberá permitir identificar, caso de haberlos, fallos y limitaciones en el tratamiento de este tipo de contenidos en la radio; así como sobre todo aquellos parámetros, consideraciones y experiencias de tipo positivo que puedan haber sido ya puestos en práctica en los programas especializados dirigidos a estos colectivos.

Fase III) A partir del análisis previo, particularmente del cualitativo y muy especialmente del referido a los programas y profesionales especializados, así como del es-

6 "Cuaderno Mayor", de Radio 5 de RNE, a cargo de Juani Loro;“Juntos paso a paso", de RNE, a cargo de Juan Fernández Vegue; "Palabras Mayores", en la actualidad en Radio Intereconomía, a cargo de Juani Loro; "La voz de los mayores”, Onda Azul Radio de Málaga, presenta Antonio Guadamuro; "Segundo Hogar", Radio Gorbea de Miranda de Ebro (Burgos); "Nuestros mayores", Radio Encuentro en Madrid, presenta Esperanza Suárez; "Mayores en las Ondas", Ràdio Cabrera de Mar (Barcelona) y Radio 7 de Alicante, a cargo de Amparo Suay. Aunque su programa finalizó en septiembre de 2007, se entrevistará igualmente, dados los 20 años que duró la emisión de su programa pionero, a Loles Díaz Aledo, responsable de "El club de la vida", de RNE. 
tudio de otras propuestas previas de recomendaciones sobre esta misma cuestión (AAVV, 2011; EAPN, 2012), se elaborará unas recomendaciones - un libro de estilo básico- sobre el tratamiento radiofónico de los mayores y la vejez, orientado al objetivo final de su contribución a un envejecimiento activo. Estas recomendaciones se harán:

III.a) tanto en un sentido preventivo: en qué medida los medios deben evitar un tratamiento incorrecto, estereotipado o perjudicial de los mayores (vulnerabilidad)

III.b) como en sentido proactivo: de qué manera los medios pueden contribuir a promover el envejecimiento activo (empoderamiento) y favorecer una ciudadanía mediática y real más efectiva de las personas mayores.

Entendemos que esta contribución puede ser de gran utilidad por las razones antes apuntadas: por la evolución demográfica de las personas mayores y su creciente importancia; por el nuevo espacio vital que se abre en las vidas temporalmente más amplias de los mayores; y como reflejo asimismo del cambio hacia una ética de la comunicación más amplia que asuma la responsabilidad del papel de los medios en relación a este colectivo en particular.

\section{Fase inicial: análisis cuantitativo de la presencia temática de la vejez en ma- gazines de la franja matinal}

De los aspectos que señalábamos en el punto anterior, traemos aquí a colación los resultados ya obtenidos del análisis cuantitativo de la presencia de los mayores y los contenidos referidos a ellos en la radio, tanto del tiempo conjunto como por áreas temáticas.

Identificamos la presencia y temas referentes a la vejez y el envejecimiento en una muestra que comprende el periodo entre el 10 y el 16 de febrero de 2014. Se trata de un período elegido de forma aleatoria, de modo que los resultados puedan considerarse estándar. El estudio empírico comprende un total de 122 horas y 30 minutos de la programación de lunes a viernes de cuatro emisoras de radio; y 31 horas de las mismas emisoras de sábado y domingo; con un total 153 horas y 30 minutos. Tomamos para la muestra los magacines de la franja matinal de la Cadena SER, Onda Cero, Cadena COPE y RNE, pues son los que mayor audiencia tienen?

En cuanto al tamaño de la muestra, Krippendorff (1994) señala que no existe una medida preestablecida, y que si todas las unidades muestrales son idénticas, una muestra constituida por una sola unidad será satisfactoria; pero si, por el contrario, en la lista de unidades hay algunos casos extraordinarios, infrecuentes y significativos, la muestra tendrá que ser ampliada. Además, mantiene que, aunque cada unidad adicional de la muestra aumenta la dificultad del análisis, hay un punto en el que ningún aumento posterior mejorará apreciablemente la generalizabilidad de los resultados. En este sentido, el autor se refiere a los trabajos realizados por Stempel, en 1952, que "comparó muestras constituidas por seis, doce, dieciocho, veinticuatro y cuarenta y ocho nú-

${ }^{7}$ Los datos según resumen del Estudio General de Medios (EGM). Por franjas horarias es la de la mañana la que registra más oyentes pues representa uno de los primetime de la radio, junto a la del mediodía y la medianoche. V. estos datos en http://www.aimc.es/-Datos-EGMResumen-General-.html [Fecha de consulta: 3 de febrero de 2014]. 
meros de un periódico con los números aparecidos a lo largo de todo un año, y comprobó, utilizando como medida la proporción media de materias tratadas, que el aumento del tamaño de la muestra más allá de doce no producía resultados significativamente más precisos" (Krippendorff, 1994: 101).

Ya en cuanto a los resultados de este estudio, se observa una presencia del 4,65\% de la temática referente a envejecimiento, teniendo en cuenta los tiempos totales de los cuatro magacines de lunes a domingo (v. Gráfico 1)

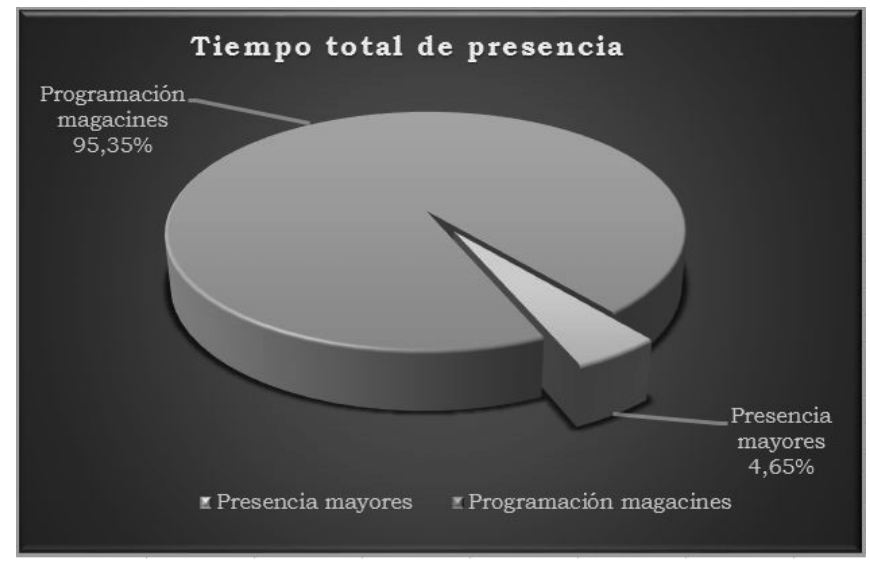

Gráfico 1. Tiempo total de presencia de la temática de mayores en los magacines de radio de lunes a domingo (Elaboración propia)

Observamos a través de esta muestra que la temática relativa a mayores de 65 años arroja cifras bajas en estos magacines de la franja matinal de la radio generalista española. Su presencia es escasa, teniendo en cuenta el porcentaje de población mayor de 65 años, que se sitúa actualmente en el 18,2\%, un porcentaje que además, como hemos visto, tiende a aumentar. Destaca por tanto, para comenzar, una notable desproporción entre su presencia en radio y el porcentaje de población del que hablamos: $4,65 \%$ frente a $18,2 \%$, es decir 13,55 puntos de desequilibrio.

El estudio similar más reciente sobre el mismo tema, si bien para el caso de la televisión, procedente de un ámbito geográfico asimilable al nuestro, arroja datos similares aunque algo superiores al porcentaje que obtenemos en nuestro estudio referido a la radio. Así, el Consell de l'Audiovisual de Catalunya (2013) analizó la representación de las personas mayores en las emisiones de televisión en Cataluña durante el primer trimestre del año $2012^{8}$. Según su estudio, las personas mayores tienen una presencia en pantalla diferente según la tipología de programación: el 10,3\% de los in-

${ }^{8}$ El estudio establece unidades y herramientas de análisis diversas en función de la tipología de los programas: teleinformativos, otros programas de no-ficción y espacios de ficción. Se analiza la presencia (en términos de frecuencia y de tiempos de palabra), la distribución por sexos, el origen percibido, la inmigración y la diversidad funcional. La segunda y tercera parte incluye la información sobre la presencia de las personas mayores en debates, entrevistas y programas de ficción. En conjunto se analizan 4.431 insertos emitidos en teleinfor- 
sertos presentes en los teleinformativos analizados corresponden a personas mayores, el 13,6\% de los debates y entrevistas, y un 7,0\% de los espacios de ficción, con una media no ponderada de 10,3\% (v. Gráfico 2). Es decir, 5,65 puntos por encima de la radio, pero todavía con 7,9 puntos de desequilibrio respecto al porcentaje de población española mayor de 65 años.

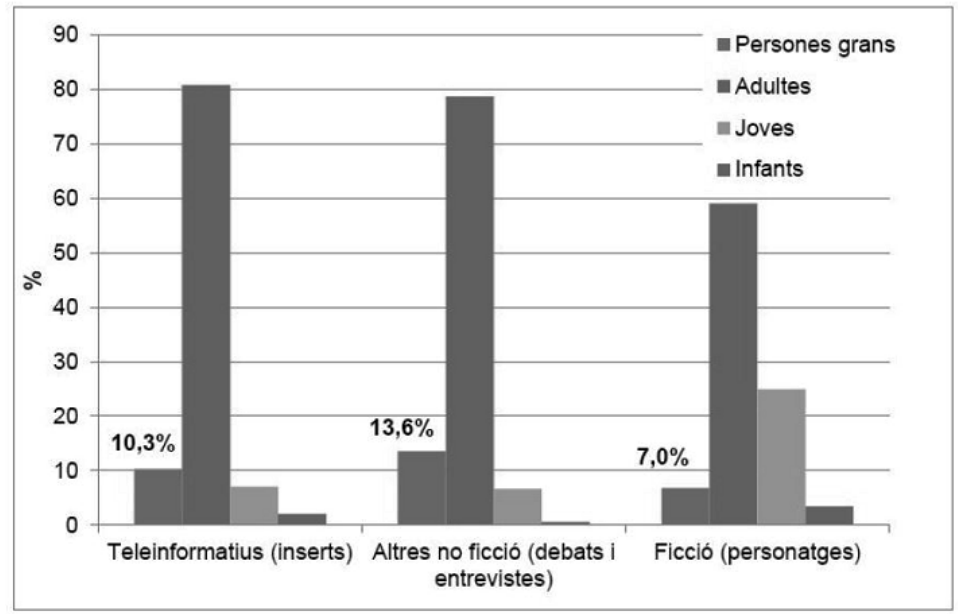

Gráfico 2. Presencia de las personas mayores en la programación televisiva emitida en Cataluña (Fuente: CAC, 2013)

Dentro del análisis de la aparición de la temática relativa a mayores de 65 años en los magacines de la franja matinal de la radio generalista también se ha realizado la comparativa entre la programación de lunes a viernes y de sábado a domingo. Teniendo en cuenta el tiempo total de duración de los respectivos espacios, hay un incremento significativo de la presencia de la temática de envejecimiento durante el fin de semana $\left(10^{\prime} 15 \%\right)$ comparado con el resultado de entre semana (3'26\%). Esto seguramente debe correlacionarse con la mayor inclinación hacia los contenidos sociales en los magacines de sábado y domingo en comparación con los emitidos de lunes a viernes.

También se ha comparado el tiempo de presencia de la temática de mayores a partir de los resultados obtenidos en cada una de las 4 cadenas de radio analizadas. Se observa que hay una mayor presencia de este contenido en los magacines de RNE, quizás porque en el ejercicio de su función de servicio público presta mayor atención a la información referente a este colectivo. De hecho, en la radio pública casi se triplica la cifra de atención a esta temática respecto a la emisora que menos atención le presta, que es Onda Cero.

mativos, 2.690 intervenciones en debates o entrevistas que se corresponden a 783 emisiones de programas y 357 personajes de ficción de 169 episodios.

La muestra incluye los informativos de TV3, TVE en Cataluña, Canal 8tv y Barcelona TV durante el primer trimestre del año 2012; la programación durante este período de TV3, Canal 33 y Canal 8tv sobre debates y entrevistas y a la de TV3, Canal 33, Canal 3XL y Canal 8tv para la ficción de producción propia. 
A los magacines de RNE le siguen en presencia de esta temática, por este orden, los de la Cadena SER, Cadena COPE y, finalmente, Onda Cero (v. Gráfico 3).

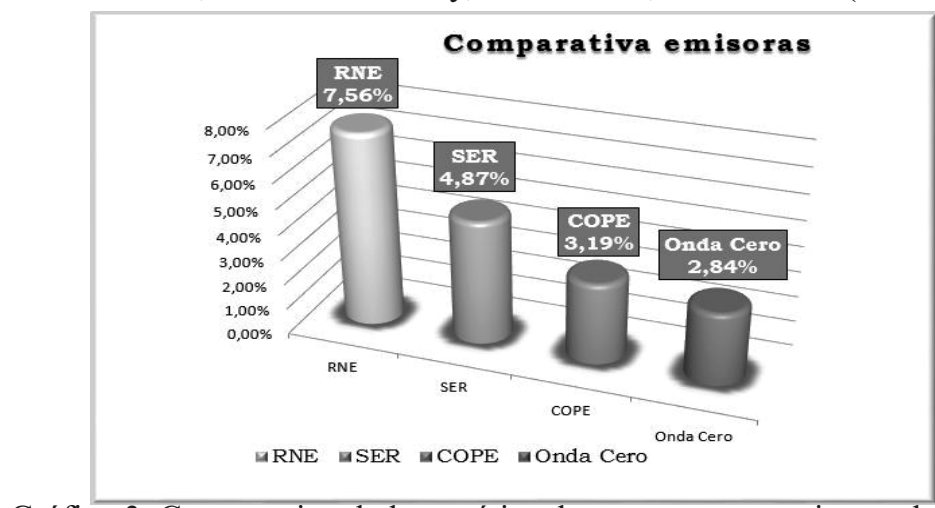

Gráfico 3. Comparativa de la temática de mayores por emisoras de radio

(Elaboración propia)

Junto a la cuantificación de la aparición de la temática estudiada se ha identificado también la variedad de temas en los que se detecta la presencia de este contenido. En conjunto, para todo el espectro de tiempo y de emisoras analizados, se ha recogido un total de 155 piezas o intervenciones difundidas en reportajes, entrevistas y resumenes de noticias. Estas piezas se han correlacionado con los siguientes 13 temas: Cultura, Política, Religión, Sucesos, Economía, Social, Envejecimiento activo, Anécdotas y curiosidades, Historia, Salud, Ciencia, Gastronomía y Literatura9 ${ }^{9}$. Se presenta una comparativa por temáticas, reflejándose el número de piezas o intervenciones referidas o cada una de los temas.

Del total de emisoras, la relación entre el colectivo senior y la temática Cultura es la que registra la presencia más intensa. La temática Cultura concentra 51 entradas, seguida de Política con 31, y de Religión con 19 (v. Gráfico 4).

En cuanto a distribución de contenidos por temáticas, según emisoras, por poner unos ejemplos de estas tres temáticas más abordadas en la muestra, se observa lo siguiente. Con relación a Cultura, cabe señalar que durante esta semana, sobre todo a principios de la misma, los magacines matinales ofrecen a sus oyentes amplia información sobre la Gala de los Premios Goya 2014, y hay muchas referencias a la actriz Terele Pávez, de 74 años, que recibió su primer Goya como mejor actriz de reparto por Las Brujas de Zugarramurdi, y a Juan Carrión, de 89 años, el profesor de inglés que inspiró otra de las películas triunfadoras, Vivir es fácil con los ojos cerrados.

9 A la hora de aplicar a los contenidos una tipología de temas, hemos preferido basarnos en una clasificación temática propia. En el estudio del Consell de l'Audiovisual de Catalunya (CAC, 2013) ya mencionado se establece una clasificación que contiene 13 temáticas referentes a la intervención de las personas mayores en televisión: Economía y negocios, Sociedad, Crónica política, Arte y Cultura, Conflictos sociales, Medio ambiente, Sanidad, Educación y enseñanza, Trabajo, Medios de comunicación y telecomunicaciones, Otros temas, Ciencia y tecnología, y Deportes. Observamos que esta clasificación y la elaborada para el presente estudio tiene 6 temas en común: Economía, Sociedad, Política, Cultura, Sanidad y Ciencia. 


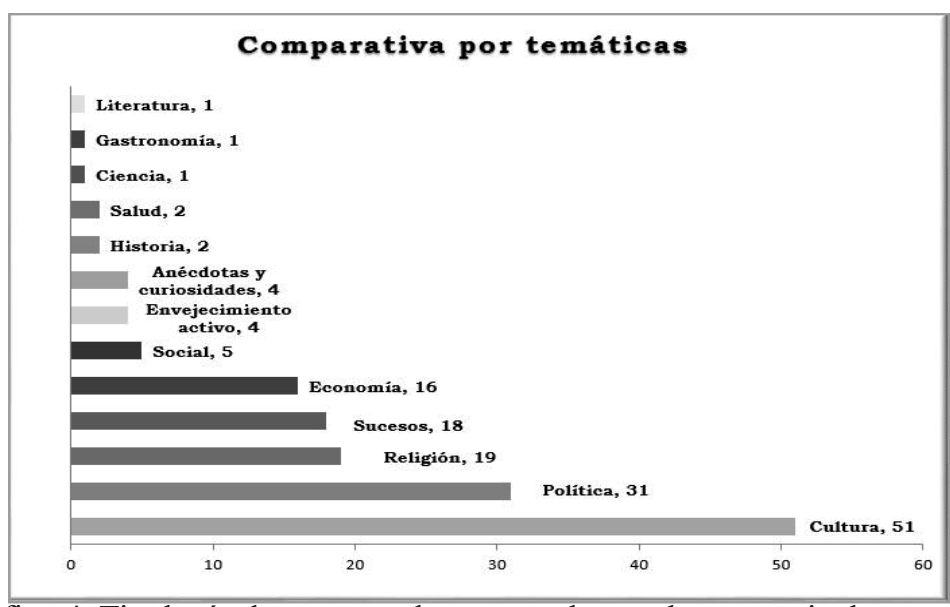

Gráfico 4. Tipología de temas en los que se detecta la presencia de contenido relacionado con mayores (Elaboración propia)

Además, hay alguna mención al vínculo entre la radio y los mayores, por el Día Mundial de la Radio (13 de febrero), pero motivada por las intervenciones telefónicas de los oyentes. Se trata del testimonio de dos mayores que reconocen la importancia de la radio en su vida: "La radio fue mi escuela, tengo 73 años", "Mi recuerdo es sobre todo de mi niñez, tengo 77 años; la radio nos reunía en toda la mesa", ambos emitidos en RNE. Estos ejemplos vienen a corroborar además lo que señalábamos más arriba: a) tanto la importancia de la radio para un sector relevante del colectivo de mayores (v. supra 3.1.), como se refleja bien en estos dos testimonios; como b) la escasa atención que se presta a este colectivo de destinatarios (v. supra 3.2.), incluso en una fecha que podría haber sido especialmente propicia para ello.

También hay numerosas referencias a Shirley Temple, "la eterna niña prodigio" del cine, debido a que fallece durante esta semana a los 85 años de edad. Observamos aquí además alguna alusión directa a las personas mayores que en esos momentos están al otro lado del receptor. "Para muchos de los mayores que nos estén escuchando, les recordará a parte de su infancia, a los años 30, que fue un mito", en referencia a Shirley Temple por su fallecimiento (Cadena Cope, 12 de febrero).

Por otra parte, la presencia en Política se debe mayoritariamente a las referencia a políticos y no a temas específicos que pudieran afectar a este colectivo. Así, las numerosas referencias a Giorgio Napolitano, presidente de la República de Italia, de 89 años de edad, por una crisis de gabinete, y también al político Silvio Berlusconi, de 78 años. Se observan menciones a Giorgio Napolitano, por ejemplo, en el magacín del 12 de febrero de la Cadena Ser. También, en la misma línea, en el magacín del 14 de febrero de Onda Cero, en el de la Cadena Cope, o en el de RNE de este mismo día. RNE hace referencia, por ejemplo, en su programa de la franja matinal de este día al "anciano Napolitano".

La temática de Religión ocupa el tercer lugar en cuanto a presencia en la muestra. Durante esta semana se ofrece amplia cobertura al dato de que se cumple un año de la renuncia de Benedicto XVI, de 87 años, al Papado. También hay menciones al ac- 
tual Papa Francisco, de 77 años. La Cadena Cope, por ejemplo, en su magacín de la franja matinal del 11 de febrero, se refiere a este tema en varias ocasiones. En uno de sus repasos informativos, el locutor señala que "Benedicto XVI adujo razones de edad", que "había mucho por hacer y le fallaban las fuerzas". Este mismo día, también en el repaso en titulares de las noticias más importantes del día, en el magacín matinal de la Cadena Cope, el locutor señala que Benedicto XVI asegura a quien vive con él que "es bello ser anciano".

La dedicación, en número de piezas o intervenciones referidas a estos asuntos, por estaciones de radio muestra que la cadena que más intervenciones destina al contenido de Cultura es RNE, con 23 entradas. En RNE se contabilizan, además, 4 piezas referidas a Política y 3 a Religión.

Por su parte es Onda Cero, con 16 piezas, la emisora que más segmentos informativos dedica a la temática de Política. Además en esta misma cadena, se anotan 7 intervenciones referidas a la temática de Cultura, y no dedica ninguna al contenido Religión. Con relación a este tema es Cadena COPE quien más entradas realiza, con un total de 14 piezas registradas. La emisora, perteneciente a la Conferencia Episcopal, emite además 12 piezas o intervenciones con la temática de Cultura y 5 piezas con contenido de Política. Por último, en la Cadena SER se consignan 9 piezas referidas a la temática de Cultura, 6 a Política y 2 a Religión.

Si contemplamos los resultados reseñados en el trabajo de investigación realizado por el Consell de l'Audiovisual de Catalunya, y únicamente a título informativo dadas las diferencias existentes entre ambos estudios, en primer lugar se encuentra la temática de Economía y Negocios, seguida de Sociedad y de Crónica política (v. Gráfico 5). En este sentido, únicamente se produce una coincidencia con la temática de Política, que se sitúa entre los 3 primeros puestos, en ambas clasificaciones. En el caso del estudio catalán, el tema de Política ocupa el tercer lugar, y en el presente análisis aparece en un segundo puesto.

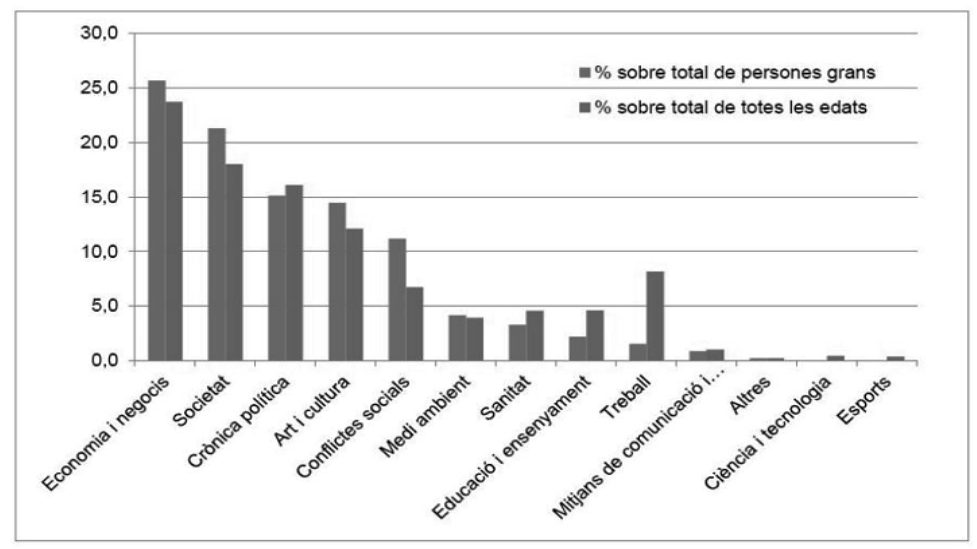

Gráfico 5. Porcentaje de insertos según la temática de la información en la televisión emitida en Cataluña (Fuente: CAC, 2013) 
Según se desprende de los resultados, la relación entre las personas mayores y la radio, no parece ser la adecuada a nivel cuantitativo, más si se tiene en cuenta el porcentaje ya relevante y en aumento creciente de los mayores en la sociedad. Su presencia en todos los ámbitos de la vida y su condición de ciudadanos de pleno derecho debería reflejarse en una mayor presencia cuantitativa. Lograrlo es tarea que compete a las propias personas mayores, pero también a la radio como medio de comunicación, particularmente a sus profesionales mediante la oportuna sensibilización al respecto, objetivo al que aspira la presente investigación.

\section{Conclusiones}

Esta primera aproximación al tratamiento de la vejez en los medios y más específicamente en la radio nos permite obtener algunas conclusiones.

i) La posición central de los medios en nuestras sociedades obliga a ampliar la ética de la comunicación más allá de los deberes fundamentales de los primeros códigos deontológicos. Esto requiere abordar el tratamiento que los medios dan a diferentes colectivos que sufren una posición de vulnerabilidad en nuestra sociedad.

ii) Uno de estos colectivos podría ser el de la tercera edad, cuyo peso demográfico está creciendo notablemente y lo hará aún más en las próximas décadas, lo que también tiene que ver con el alargamiento de este período de la vida humana. Se ha planteado un cambio de paradigma para abordar el reto de la calidad de vida y la participación que supone esta prolongación de la vejez, el del envejecimiento activo. Los medios deben atender a estos cambios y a los planteamientos de este nuevo paradigma; la radio puede ser especialmente útil aquí por sus características de accesibilidad, familiaridad de uso, cercanía, proximidad afectiva y complicidad.

iii) Sin embargo, los resultados obtenidos en la investigación ya realizada indican una baja representación del colectivo de mayores incluso en un soporte, un formato y una franja horaria -los magazines radiofónicos matinales- que podrían hacer pensar en una mayor presencia. Se produce así un desequilibrio de 13,55 puntos entre el porcentaje de población mayor de 65 años $(18,2 \%)$ y la presencia de la vejez en los programas de radio analizados en las principales emisoras de radio del país (4,65\%). Lo que plantea un primer reto de una mayor visibilización de este colectivo.

Esta mayor visibilización -como anticipo de las siguientes Fases de nuestro trabajo- debería orientarse además hacia la promoción del envejecimiento activo, para lo cual las recomendaciones que en su día se sigan de este estudio en sus fases más avanzadas deberán resultar de especial utilidad para gestores y profesionales de la radio.

\section{Referencias bibliográficas}

AA.VV. (2011): Las personas mayores y los medios de comunicación en Bizkaia. Bilbao, Departamento de Acción Social de la Diputación Foral de Bizkaia. http://www.bizkaia.net/home2/Archivos/DPTO3/Temas/Pdf/medios_WEB_ca.pdf [Consulta: 27 de octubre de 2014].

ABDULLAH, Boushra \& WOLBRING, Gregor (2013): "Analysis of Newspaper Coverage of Active Aging through the Lens of the 2002 World Health Organization Active Ageing Report: A Policy Framework and the 2010 Toronto Charter for Physical 
Activity: A Global Call for Action". International Journal of Environmental Research and Public Health, $n^{\circ} 10$, pp. 6799-6819. http://www.ncbi.nlm.nih.gov/pmc /articles/PMC3881142/ [Consulta: 2 de diciembre de 2014]

ABELLÁN, Antonio y PUJOL, Rogelio (2015): "Un perfil de las personas mayores en España, 2015. Indicadores estadísticos básicos". IMSERSO (Informes Envejecimiento en red $\mathrm{n}^{\mathrm{o}} 10$ ). http://envejecimiento.csic.es/documentos/documentos /enred-indicadoresbasicos 15.pdf [Consulta: 2 de febrero de 2015]

ACOSTA, Maribel; BADÍA, Ana T.; y COSTALES, Zenaida (2014): "Radio pública. Apuntes conceptuales". Más Poder Local. Revista en Comunicación Política, $\mathrm{n}^{\circ}$ 21, pp. 45-50.

AZNAR, Hugo (2000): Ética y periodismo. Barcelona, Paidós Comunicación.

AZNAR, Hugo (2005): Ética de la comunicación y nuevos retos sociales. Barcelona, Paidós Comunicación.

BALSEBRE, Armand (1994): El lenguaje radiofónico. Madrid, Cátedra Signo e Imagen.

BORNSTEIN, David (2011): «Why ‘Solutions Journalism' Matter, Too», en New York Times, 20 de diciembre. http://opinionator.blogs.nytimes.com/2011/12/20/ whysolutions-journalism-matters-too/ [Consulta: 21 de septiembre de 2014]

CARBAJOSA, Ana (2014): "El despilfarro de la experiencia”, en El País, 16 de noviembre, sección Domingo, p. 2.

CEBRIÁN, Mariano (1994): Información radiofónica. Mediación técnica, tratamiento y programación. Madrid, Editorial Síntesis.

CSIC (2010): Informe de la I+D+i sobre envejecimiento. Madrid, Fundación General del CSIC http://www.fgcsic.es/envejecimiento/upload/recursos_documentos.11.ccqDescarga.Informesobreenvejecimiento.pdf [Consulta: 29 de septiembre de 2014]

CONSELL DE L'AUDIOVISUAL DE CATALUNYA (CAC) (2013): La representació de la gent gran a la televisió. Informe sobre la diversitat $i$ la igualtat. Barcelona, CAC.

DÍAZ, Rosa (2012): Informe 2010 Las Personas Mayores en España. Datos Estadísticos Estatales y por Comunidades Autónomas. Tomo I. IMSERSO, Observatorio de Personas Mayores. http://www.imserso.es/InterPresent1/groups/imserso/documents/binario/22023_inf2010pm_v1.pdf [Consulta: 20 de diciembre de 2013].

EUROPEAN ANTIPOVERTY NETWORK (EAPN) (2012): Guía de estilo para periodistas sobre Personas Mayores. Madrid, EAPN.

FERNÁNDEZ-BALLESTEROS, Rocío (2002): Envejecer bien: ¿Qué es y cómo lograrlo? Madrid, Ediciones Pirámide.

GIRÓ, Joaquín (2006): Envejecimiento activo, envejecimiento en positivo. España, Universidad de La Rioja. 
HARWOOD, Jake (2007): Understanding Communication and Aging. London, Sage. INE (2014a): "Movimiento Natural de la Población (Nacimientos, Defunciones y Matrimonios). Indicadores Demográficos Básicos. Datos Provisionales. Año 2013". Madrid, INE (Nota de prensa). http://www.ine.es/prensa/np851.pdf [Consulta: 5 de julio de 2014].

INE (2014b): "Proyección de la Población de España 2014-2064". Madrid, INE (Nota de prensa). http://www.ine.es/prensa/np870.pdf [Consulta: 5 de noviembre de 2014].

IMSERSO (2011): Envejecimiento activo. Libro blanco. Madrid, IMSERSO.

KRIPPENDORFF, Klaus (1994). Metodología de análisis y contenido. Teoría y práctica. Barcelona, Paidós.

LAITILA, Tiina (1995): "Journalistic Codes of Ethics in Europe". European Journal of Communication, $\mathrm{X}, \mathrm{n}^{\circ} .4$, pp. 527-544.

LIPPMANN, Walter ([1920] 2011): Libertad y prensa. Madrid, Tecnos.

LÓPEZ NOGUERO, Fernando (2001): "Los medios de comunicación en la educación social: el uso de la radio". Comunicar, no 16, p.141-148.

MERAYO, Arturo (1992): Para entender la radio: Estructura del proceso informativo radiofónico. Salamanca, Publicaciones Universidad Pontificia de Salamanca.

SARTORI, Giovanni (1998): "La opinión teledirigida". Claves de la razón práctica, $\mathrm{n}^{\circ} 49$, pp. 2-7.

TRAVERS, Catherine \& BARTLETT, Helen P. (2011): "Silver Memories: Implementation and evaluation of a unique radio program for older people". Aging \& Mental Health, Vol. 15, $\mathrm{n}^{\mathrm{o}}$ 2, pp. 169-177. http://www.tandfonline.com/doi /full/10.1080/13607863.2010.508774 [Consulta: 2 de noviembre de 2014].

UNESCO (2013): The Principle of Respect for Human Vulnerability and Personal Integrity. París, Unesco.

VERA ARANDA, José Luis (2005): Televisión y telespectadores. Huelva, Universidad de Huelva.

ZAMARRÓN, María Dolores (2007): "Envejecimiento activo". INFOCOP, núm. 34, pp. 7-9.

Hugo Aznar es Doctor en Filosofía y Profesor Agregado de Ética de la Comunicación e Historia del Pensamiento Político. Responsable de la Línea de Investigación Democracia deliberativa, comunicación y ciudadanía. Departamento de CC. Políticas, Ética y Sociología Universidad. Universidad CEU Cardenal Herrera (Valencia)

Ángels Álvarez Villa es Doctora en CC. de la Comunicación, Profesora de Radio y Directora de Radio CEU. Miembro del Grupo de Investigación sobre 
Discapacidad y Comunicación (GIDYC). Departamento Comunicación Audiovisual, Publicidad y Tecnología de la Información. Universidad CEU Cardenal Herrera (Valencia)

Amparo Suay Madrid es Licenciada en Periodismo y Doctoranda en el Programa de Comunicación de la Universidad CEU Cardenal Herrera. Directora del programa radiofónico "Mayores en las Ondas" de la Fundación Salud y Comunidad (FSC), entidad especializada en gestión de servicios para personas mayores. Fundación Salud y Comunidad (FSyC) 